\title{
Frequency Domain Approach for Hopf Bifurcation Analysis in a Single Mode Laser Model with Time Delay
}

\author{
Changjin Xu \\ Faculty of Science, Hunan Institute of Engineering, Xiangtan 411004, China
}

$\&$

Department of Mathematics, Central South University, Changsha 410083, China

Tel: 86-732-268-0745 E-mail: xcj403@126.com

Maoxin Liao

Department of Mathematics, Central South University, Changsha 410083, China

$\&$

School of Mathematics and Physics, Nanhua University, Hengyang, 421001, China

The research is supported by National Natural Science Foundation of China (No. 10771215)and the Scientific Research Initializing Foundation of Hunan Institute of Engineering (0744).

\begin{abstract}
IIn this paper, using frequency domain approach, a single mode laser model with delay is investigated. By choosing the delay $\tau$ as a bifurcation parameter, we show that Hopf bifurcation can occur when $\tau$ passes a sequence of critical values. This means that a family of periodic solutions bifurcate from the equilibrium when the bifurcation parameter exceeds a critical value. Some numerical simulations are given to justify the theoretical analysis results. The approach used in this paper is an excellent supplement of previous known ones of Hopf bifurcation analysis.
\end{abstract}

Keywords: Single mode laser model, Hopf bifurcation, Stability, Frequency domain approach, Nyquist criterion

\section{Introduction}

In optics, the laser is a typical example which produces the phenomenon of self-organization. It is currently one of the focus of research in laser field. In recent year, a lot of laser models that obtain self-organization phenomena have been proposed. In 1998, Lu et al. (Lu et al., 1998) investigated the chaotic behavior of the following single mode laser system:

$$
\left\{\begin{aligned}
\frac{d x_{1}(t)}{d t} & =-a x_{1}(t)+x_{2}(t) \\
\frac{d x_{2}(t)}{d t} & =-b x_{2}(t)+x_{1}(t) x_{3}(t) \\
\frac{d x_{3}(t)}{d t} & =c-x_{3}(t)-x_{1}(t) x_{2}(t) .
\end{aligned}\right.
$$

The specific meaning of model (1), one can see (Lu et. al, 1998). Taking into account that there is a certain time delay during the input signal and the transmission signals, the dynamic behavior of the system not only is affected by the current state of the system, but also the past state of the system, i.e., there exists inherent lag in the system. Based on this view of point, in this paper, we will investigate the following single mode laser system with delay:

$$
\left\{\begin{aligned}
\frac{d x_{1}(t)}{d t} & =-a x_{1}(t)+x_{2}(t), \\
\frac{d x_{2}(t)}{d t} & =-b x_{2}(t)+x_{1}(t-\tau) x_{3}(t-\tau), \\
\frac{d x_{3}(t)}{d t} & =c-x_{3}(t)-x_{1}(t-\tau) x_{2}(t-\tau) .
\end{aligned}\right.
$$

In this paper, we investigate Hopf bifurcation of the system (2). It is worth pointing out that a lot of the early work on Hopf bifurcation of the delayed differential equations is used the state-space formulation for delayed differential equations, known as the "time domain" approach. Yet, there is another interesting formulation for studying delayed differential equations in the literature. This alternative representation applies the familiar engineering feedback systems theory and methodology: an approach described in the "frequency domain"- the complex domain after the standard Laplace transforms have been taken on the state-space system in the time domain. The frequency domain approach was initiated and developed by Allwright (Allwright David J., 1977), Mees and Chua (Mees Alistair I. \& Chua Leon O., 1979), and then Moiola and Chen (Moiola Jorge L. \& Chen Guanrong, 1993, 1996). This new methodology has some advantages over the classical time-domain methods. A typical one is its pictorial characteristic that utilizes advanced computer graphical capabilities thereby bypassing quite a lot of profound and difficult mathematical analysis.

In this paper, we will devote our attention to finding the Hopf bifurcation point for models (2). And the main methodology of study is by means of the frequency-domain approach. It is found that if the coefficient $\tau$ is used as a bifurcation 
parameter, then Hopf bifurcation occurs for the model (2). This means that a family of periodic solutions bifurcates from the equilibrium when the bifurcation parameter exceeds a critical value. Some numerical simulations are given to justify the theoretical analysis results. To the best of our knowledge, it is the first time to deal with the research of Hopf bifurcation of the model (2) by the frequency-domain approach.

The remainder of the paper is organized as follows: in Section 2, by means of the frequency-domain approach formulated by Moiola and Chen (Moiola Jorge L. \& Chen Guanrong, 1996), the existence of Hopf bifurcation parameter is determined and shown that Hopf bifurcation occurs when the bifurcation parameter exceeds a critical value. In section 3 , some numerical simulation are carried out to verify the correctness of theoretical analysis result. Finally, some conclusions and discussions are given in section 4.

\section{Existence of Hopf bifurcation}

In model (2), we assume that the following condition

$$
\text { (H) } c>a b \text {. }
$$

holds.

It is easy to see that system (2) has a unique positive equilibrium $E_{*}\left(x_{1}^{*}, x_{2}^{*}, x_{3}^{*}\right)$, where,

$$
x_{1}^{*}=\sqrt{\frac{c-a b}{a}}, x_{2}^{*}=a x_{1}^{*}, x_{3}^{*}=a b .
$$

We can rewrite the nonlinear system (2) in a matrix form as

$$
\frac{d x(t)}{d t}=A x(t)+H(x)
$$

where $x=\left(x_{1}(t), x_{2}(t), x_{3}(t)\right)^{T}$,

$$
A=\left(\begin{array}{ccc}
-a & 1 & 0 \\
0 & -b & 0 \\
0 & 0 & -1
\end{array}\right), H(x)=\left(\begin{array}{c}
0 \\
x_{1}(t-\tau) x_{3}(t-\tau) \\
c-x_{1}(t-\tau) x_{2}(t-\tau)
\end{array}\right)
$$

Choosing the coefficient $\tau$ as a bifurcation and introducing a "state-feedback control" $u=g(y(t-\tau) ; \tau)$, where $y(t)=$ $\left(y_{1}(t), y_{2}(t), y_{3}(t)\right)^{T}$, we obtain a linear system with a non-linear feedback as follows

$$
\left\{\begin{array}{l}
\frac{d x}{d t}=A x+B u \\
y=-C x \\
u=g(y(t-\tau) ; \tau)
\end{array}\right.
$$

where

$$
B=C=\left(\begin{array}{lll}
1 & 0 & 0 \\
0 & 1 & 0 \\
0 & 0 & 1
\end{array}\right), \quad u[g(y(t-\tau), \tau)]=\left(\begin{array}{c}
0 \\
y_{1}(t-\tau) y_{3}(t-\tau) \\
c-y_{1}(t-\tau) y_{2}(t-\tau)
\end{array}\right)
$$

Next, taking Laplace transform on (4), we obtain the standard transfer matrix of the linear part of the system:

$$
G(s ; \tau)=C[s I-A]^{-1} B .
$$

Then

$$
G(s ; \tau)=\left(\begin{array}{ccc}
\frac{1}{s+a} & \frac{1}{(s+a)(s+b)} & 0 \\
0 & \frac{1}{s+b} & 0 \\
0 & 0 & \frac{1}{(s+1)}
\end{array}\right) .
$$

If this feedback system is linearized about the equilibrium $y=-C\left(x_{1}^{*}, x_{2}^{*}, x_{3}^{*}\right)^{T}$, then the Jacobian of (5) is given by

$$
J(\tau)=\left.\frac{\partial g}{\partial y}\right|_{y=\tilde{y}=-C\left(x_{1}^{*}, x_{2}^{*}, x_{3}^{*}\right)^{T}}=\left(\begin{array}{ccc}
0 & 0 & 0 \\
x_{3}^{*} e^{-s \tau} & 0 & x_{1}^{*} e^{-s \tau} \\
-x_{2}^{*} e^{-s \tau} & -x_{1}^{*} e^{-s \tau} & 0
\end{array}\right) .
$$

Set

$$
\begin{aligned}
h(\lambda, s ; \tau)= & \operatorname{det}|\lambda I-G(s ; \tau) J(\tau)| \\
= & \lambda\left[\lambda^{2}-\frac{x_{3}^{*} e^{-s \tau}}{(s+a)(s+b)} \lambda+\frac{x_{1}^{*} x_{2}^{*} e^{-2 s \tau}}{(s+a)(s+b)(s+1)}\right. \\
& \left.+\frac{x_{1}^{* 2} e^{-2 s \tau}}{(s+b)(s+1)}\right]=0 .
\end{aligned}
$$


Then, we obtain the following results by applying the generalized Nyquist stability criterion with $s=i \omega$.

Lemma 2.1. (Moiola Jorge L. \& Chen Guanrong, 1996) If an eigenvalue of the corresponding Jacobian of the nonlinear system, in the time domain, assumes a purely imaginary value $i \omega_{0}$ at a particular $\tau=\tau_{0}$, then the corresponding eigenvalue of the constant matrix $G\left(i \omega_{0} ; \tau_{0}\right) J\left(\tau_{0}\right)$ in the frequency domain must assume the value $-1+i 0$ at $\tau=\tau_{0}$.

To apply Lemma 2.1 , let $\hat{\lambda}=\hat{\lambda}(i \omega ; \tau)$ be the eigenvalue of $G(i \omega ; \tau) J(\tau)$ that satisfies $\hat{\lambda}\left(i \omega_{0} ; \tau_{0}\right)=-1+0 i$. Then

$$
\begin{aligned}
h\left(-1, i \omega_{0} ; \tau_{0}\right)= & i \omega_{0}\left[-\omega_{0}^{2}+\frac{x_{3}^{*} e^{-i \omega_{0} \tau_{0}}}{\left(i \omega_{0}+a\right)\left(i \omega_{0}+b\right)}+\frac{x_{1}^{*} x_{2}^{*} e^{-2 i \omega \tau_{0}}}{(i \omega+a)(i \omega+b)(i \omega+1)}\right. \\
& \left.+\frac{x_{1}^{* 2} e^{-2 i \omega \tau_{0}}}{(i \omega+b)(i \omega+1)}\right]=0 .
\end{aligned}
$$

Separating the real and imaginary parts, we obtain

$$
\begin{gathered}
M_{1} \cos \omega_{0} \tau_{0}+N_{1} \sin \omega_{0} \tau_{0}=-x_{3}^{*}, \\
M_{2} \cos \omega_{0} \tau_{0}-N_{2} \sin \omega_{0} \tau_{0}=-x_{3}^{*} \omega_{0},
\end{gathered}
$$

where

$$
\left\{\begin{array}{l}
M_{1}=x_{1}^{*} x_{2}^{*}+a x_{1}^{*}-\omega_{0}^{2}\left(a b-\omega_{0}^{2}-\omega_{0}^{2} a-\omega_{0}^{2} b\right), \\
N_{1}=\omega_{0} x_{1}^{*}+\omega_{0}\left(a b-\omega_{0}^{2}\right)+(a+b) \omega_{0}, \\
M_{2}=\omega_{0} x_{1}^{*}-\omega_{0}\left(a b-\omega_{0}^{2}\right)-(a+b) \omega_{0}, \\
N_{2}=x_{1}^{*} x_{2}^{*}+a x_{1}^{*}+\omega_{0}^{2}\left(a b-\omega_{0}^{2}-\omega_{0}^{2} a-\omega_{0}^{2} b\right) .
\end{array}\right.
$$

Thus, we bave

$$
\begin{aligned}
& \cos \omega_{0} \tau_{0}=-\frac{N_{2} x_{3}^{*}+N_{1} x_{3}^{*} \omega_{0}}{M_{1} N_{2}+M_{2} N_{1}}, \\
& \sin \omega_{0} \tau_{0}=\frac{M_{1} x_{3}^{*} \omega_{0}-M_{2} x_{3}^{*}}{M_{1} N_{2}+M_{2} N_{1}} .
\end{aligned}
$$

According to $\cos ^{2} \omega_{0} \tau_{0}+\sin ^{2} \omega_{0} \tau_{0}=1$, we get

$$
\left(N_{2} x_{3}^{*}+N_{1} x_{3}^{*} \omega_{0}\right)^{2}+\left(M_{1} x_{3}^{*} \omega_{0}-M_{2} x_{3}^{*}\right)^{2}=\left(M_{1} N_{2}+M_{2} N_{1}\right)^{2},
$$

By (10), we can compute the value of $\omega_{0}$ by means of Matlab software. Then from (10), we obtain

$$
\tau_{0}=\frac{1}{\omega_{0}}\left[(2 k+1) \pi-\arccos \frac{N_{2} x_{3}^{*}+N_{1} x_{3}^{*} \omega_{0}}{M_{1} N_{2}+M_{2} N_{1}}\right](k=0,1,2, \ldots) .
$$

Theorem 2.1. ( Existence of Hopf bifurcation parameter ) For system (2), if $\omega_{0}$ is positive real roots of (12), then then Hopf bifurcation point of system (2) is

$$
\tau_{0}=\frac{1}{\omega_{0}}\left[(2 k+1) \pi-\arccos \frac{N_{2} x_{3}^{*}+N_{1} x_{3}^{*} \omega_{0}}{M_{1} N_{2}+M_{2} N_{1}}\right](k=0,1,2, \ldots),
$$

where, $M_{i}, N_{i}(i=1,2)$ are defined by (9), respectively.

\section{Numerical Examples}

In this section, we give numerical simulations to verify the correctness of our computation. As an example, we consider the following system:

$$
\left\{\begin{aligned}
\frac{d x_{1}(t)}{d t} & =-0.2 x_{1}(t)+x_{2}(t) \\
\frac{d x_{2}(t)}{d t} & =-0.3 x_{2}(t)+x_{1}(t-\tau) x_{3}(t-\tau) \\
\frac{d x_{3}(t)}{d t} & =0.6-x_{3}(t)-x_{1}(t-\tau) x_{2}(t-\tau) .
\end{aligned}\right.
$$

By Theorem 2.1, we obtain $\tau_{0} \approx 0.22$. Let $\tau=0.21$, then the figures of numerical simulations are Fig.1-6. Thus we conclude that when $\tau<\tau_{0} \approx 0.22$, system (14) is asymptotically stable. Let $\tau=0.23$, then the figures of numerical simulations are Fig.7-12. Thus we conclude that when $\tau>\tau_{0} \approx 0.22$, system (14) undergoes a Hopf bifurcation occurs near the positive equilibrium. Therefore $\tau_{0} \approx 0.22$ is a supercritical Hopf bifurcation point.

\section{Conclusions and discussions}

In this paper, we investigated a class of ecological model with delay. By choosing the coefficient $\tau$ as a bifurcating parameter and analyzing the associating characteristic equation. It is found that a Hopf bifurcation occurs when the bifurcating parameter $\tau$ passes through a critical value. Considering computational complexity, the direction and the stability of the bifurcating periodic orbits for system (2) have not been studied. It is beyond the scope of the present paper and will be further investigated elsewhere in the future. 

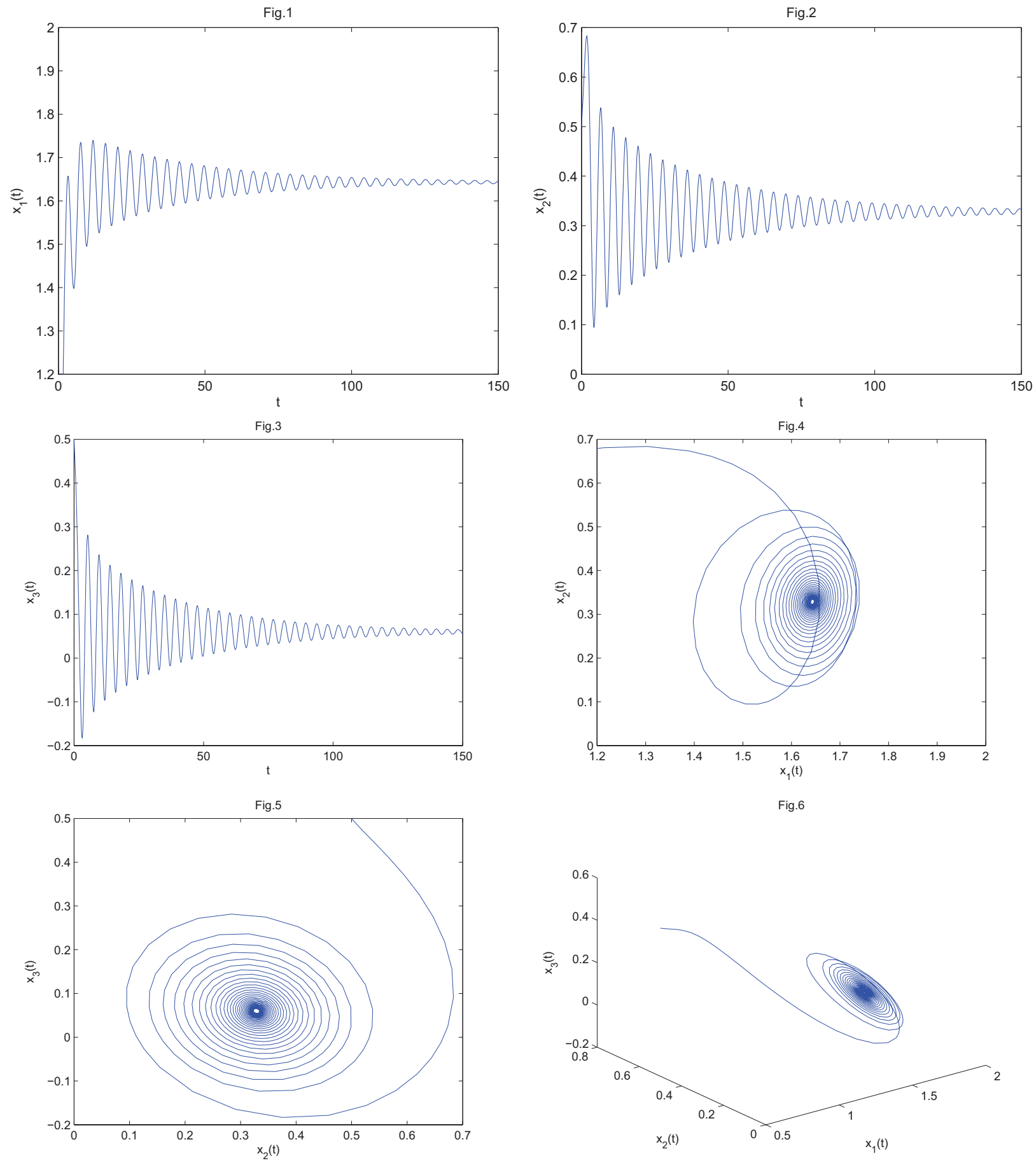

Figure 1-6. Behavior and phase portrait of system (14) with $\tau=0.21<\tau_{0} \approx 0.22$. The positive equilibrium is asymptotically stable. The initial value is $(0.5,0.5,0.5)$ 

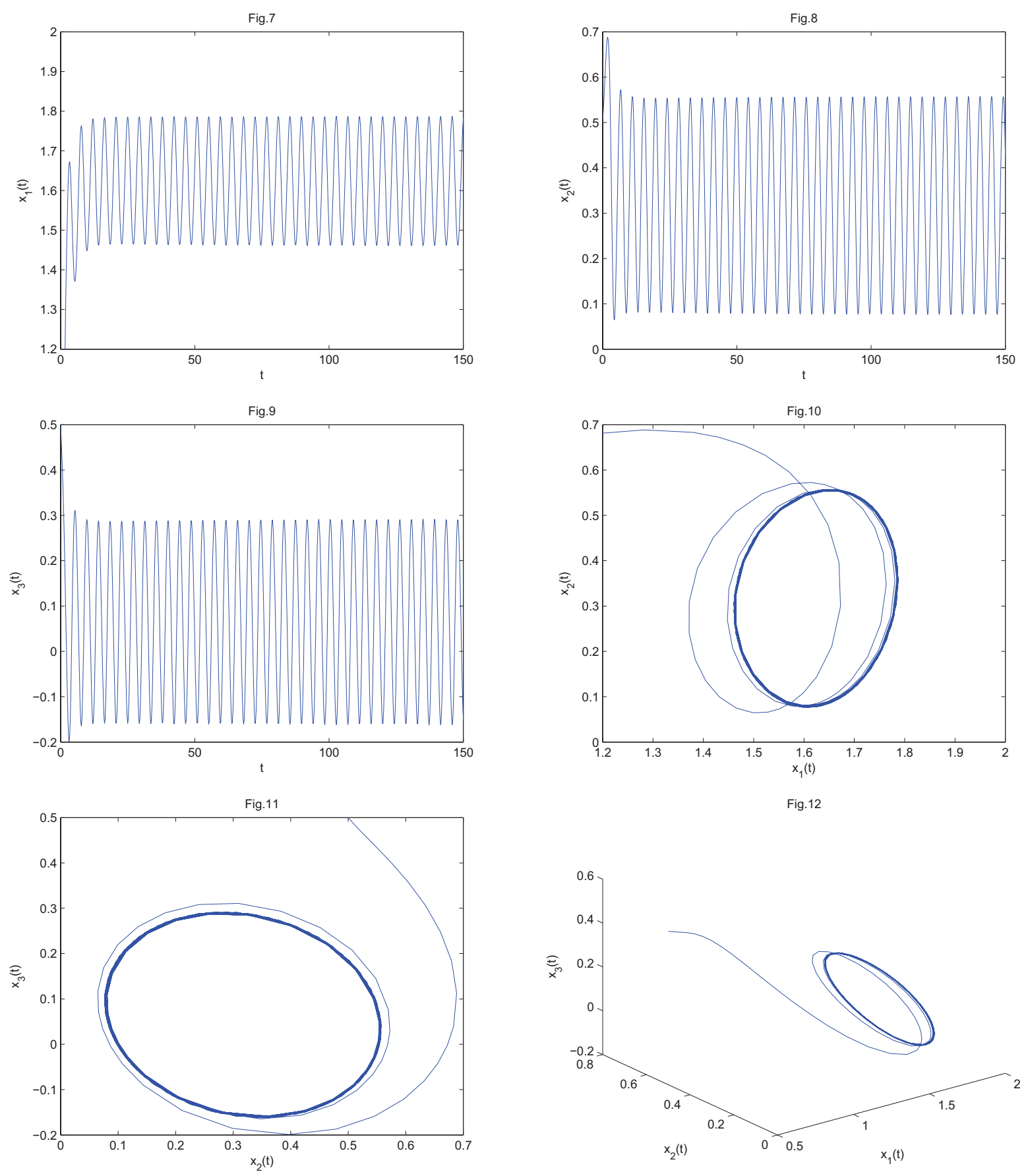

Figure 7-12. Behavior and phase portrait of system (14) with $\tau=0.23>\tau_{0} \approx 0.22$. Hopf bifurcation occurs from the positive equilibrium. The initial value is $(0.5,0.5,0.5)$

\section{References}

Allwright David J. (1977). Harmonic balance and the Hopf bifurcation theorem. Mathematical Proceedings of the Combridge Philosophical Society, No.82: p.453-467.

Li Gang. (2007). Chaos Synchronization between Single-Mode Laser Lorenz System and 3D Chaotic System. Acta Photonica Sinca, No.36(5). p.808-811. (in chinese)

Liao Xiaofeng, Li Shaowen \& Chen Guanrong. (2004). Bifurcation Analysis on a Two-neuron system with distributed delays in the frequency domainkl. Neural Networks, No.17. p.545-561.

Liao Xiaofeng \& Li Shaowen. (2003). Hopf bifurcation on a two-neuron system with distributed delays: a frequency domain approach. Nonlinear Dynamacis, No.31. p. 299-326.

Lu Ling, Li Chengren \& Chen Boqiao. (1998). Theoretical research of chaotic behavior about single mode laser. Optical Technique, No.2. p.35-43. (In chinese) 
Mees Alistair I. \& Chua Leon O. (1979). The Hopf bifurcation theorem and its applications to nonlinear oscillations in circuits and systems. Institute of Electrical and Electronics Engineers, Transcations on Circuits and Systems, No.26. p.235-254.

Moiola Jorge L. \& Chen Guanrong. (1993). Frequency domain approach to computational analysis of bifurcations and limit cycles: a tutorial. International Journal of Bifurcation and Chaos, 3: 843-867.

Moiola Jorge L. \& Chen Guanrong. (1996). Hopf bifurcation analysis: a frequency domain approach. Singapore: World Scientific. 